University of Nebraska - Lincoln

DigitalCommons@University of Nebraska - Lincoln

September 1985

\title{
A variation-perturbation method for atomic and molecular interactions. I. Theory
}

Gordon A. Gallup

UNL,ggallup1@unl.edu

J. Gerratt

Department of Theoretical Chemistry, School of Chemistry, University of Bristol, Bristol BS8 1TS, England

Follow this and additional works at: https://digitalcommons.unl.edu/physicsgallup

Part of the Physics Commons

Gallup, Gordon A. and Gerratt, J., "A variation-perturbation method for atomic and molecular interactions. I. Theory" (1985). Gordon Gallup Publications. 11.

https://digitalcommons.unl.edu/physicsgallup/11

This Article is brought to you for free and open access by the Research Papers in Physics and Astronomy at DigitalCommons@University of Nebraska - Lincoln. It has been accepted for inclusion in Gordon Gallup Publications by an authorized administrator of DigitalCommons@University of Nebraska - Lincoln. 


\title{
A variation-perturbation method for atomic and molecular interactions. I. Theory
}

\author{
G. A. Gallup a) and J. Gerratt \\ Department of Theoretical Chemistry, School of Chemistry, University of Bristol, Bristol BS8 1TS, England
}

(Received 11 February 1985; accepted 26 March 1985)

\begin{abstract}
We have developed a variation-perturbation procedure for calculating intermolecular forces. It is based on the valence bond method of constructing wave functions and involves a number of interlocking techniques and approximations that are justified by the small size of the interaction potential relative to the total energy. In this article we give an outline of the theory of our technique. We have used this procedure to determine the potential between a $\mathrm{Ne}$ atom and a rigid HF molecule. The results of this calculation are given in the next article.
\end{abstract}

\section{INTRODUCTION AND SURVEY}

The van der Waals energy between the two parts of a system is a very small fraction of the total electronic energy. Between two 1 or 2 atom systems from the first row of the Periodic Table, this fraction is, at the most, $10^{-6}-10^{-7}$. Such a physical situation would appear to be ideally suited to treatment by perturbation theory, and many studies have taken this approach. Treating the terms of the Hamiltonian connecting the two parts of the system as a perturbation leads to "exchange perturbation theory," which has been investigated thoroughly by several workers. "As it is usually formulated, this approach has inherent difficulties associated with the different symmetries possessed by the unperturbed and total Hamiltonians with respect to electron exchange. The symmetry problem leads to non-unique perturbation expansions for the wave function. Although Peierls $^{2}$ has given a very general solution to perturbation problems of this type, the considerations are quite delicate and difficulties remain.

These have led others to the calculation of van der Waals energies from total energies directly. In this case one is faced with the necessity of subtracting two large numbers with a great loss in significant figures. Nevertheless, this approach has been used for a number of systems. ${ }^{3}$ However, it has definite limitations as to the size of systems that can be treated, and recently $a b$ initio calculations have been combined with damped semiempirical long-range dispersion potentials to treat larger systems. ${ }^{4}$ In this hybrid procedure the $a b$ initio step is an SCF calculation of the supermolecule in order to determine the repulsive part of the potential. The drawback of this procedure is that large basis set superposition errors occur, and these must be removed by the counterpoise method. ${ }^{5}$ Recently, Fowler and Buckingham ${ }^{6}$ have shown that the counterpoise corrections themselves introduce new unphysical effects.

Our approach is intermediate between these others. It is based on the conviction that the small size of van der Waals energies should make it possible to formulate the problem so that they are, in some sense, easier to calculate than total energies. We start with a variational calculation based upon valence bond functions, which are eminently suited to dis-

\footnotetext{
a) On leave during the 1983-84 academic year from the University of $\mathrm{Ne}$ braska under the auspices of the S.E.R.C., United Kingdom and University of Nebraska Research Council. Permanent address: Department of Chemistry, University of Nebraska, Lincoln, Nebraska 68588.
}

cussing intermolecular forces. ${ }^{7}$ The Hamiltonian matrix is determined with the exact Hamiltonian (excepting the approximations discussed below) and the full antisymmetry of the system so there are no problems of the sort arising in exchange perturbation theory. Using matrix partitioning techniques and numerical perturbations, an implicit expression for the interaction potential itself may be written down. This still contains differences between fairly large numbers, but these now cause no difficulty since they are present in a form where there is a systematic cancellation of certain errors associated with inadequacies in the wave functions of the subsystems.

Another part of our procedure is the optimization ${ }^{8}$ of excited orbitals for the efficient description of induction and dispersion using an adaptation of the pseudo-state polarizability technique devised by Burke and Robb. ${ }^{9}$ For the basis used, our adaptation produces the minimal number of orbitals that are required in the calculation, and the resulting economy in total numbers of configurations is an important aspect of our technique.

As a further part of our general procedure we approximate some of the atomic integrals and some of the Hamiltonian matrix elements in ways expected to have general validity for problems of intermolecular forces. In addition, still other matrix elements are subjected to a semiempirical adjustment. In the following sections we give details of all parts of this procedure.

\section{AN IMPLICIT EQUATION FOR THE POTENTIAL}

Consider a system of two interacting closed-shell ground state subsystems $a$ and $b$ that may be either atoms or molecules. We assume we have Hartree-Fock (HF) orbitals for each of the subsystems, and these are used to construct an $n$-electron VB basis for the composite system. The principal (approximate ground state) function is

$$
\phi_{0}=A\left[u_{1}^{a} \cdots u_{m}^{a} u_{1}^{b} \cdots u_{n}^{b}\right],
$$

the antisymmetrized product of the occupied spin orbitals of $a$ and $b$. We carry out a configuration interaction $(\mathrm{CI})$ treatment using, as additional functions, those obtained from various excitations from the orbitals of $\phi_{0}$. The correct choice of these functions is important for accurate intermolecular potential calculations and this is discussed below.

In the standard way, the basis functions we have constructed $\phi_{i}, i=0,1, \ldots, N$, give Hamiltonian and overlap ma- 
trices, $H(\rho)$ and $S(\rho)$ that are functions of the geometry of the system $\rho$. The lowest eigenvalue of this matrix system gives the interaction energy. Specifically, the ground state energy is given by

$$
\left[H(\rho)-E_{0}(\rho) S(\rho)\right] C_{0}(\rho)=0 .
$$

However, we seek the potential of the interaction:

$$
V(\rho)=E_{0}(\rho)-E_{0}(\infty) \text {. }
$$

In Eq. (2) $E_{0}(\infty)$ is the lowest eigenvalue of Eq. (1) when $a$ and $b$ are infinitely separated.

There is a matrix that diagonalizes $H$ and $S$ at each geometry,

$$
\begin{aligned}
& M(\rho)^{\dagger} H(\rho) M(\rho)=E(\rho), \\
& M(\rho)^{\dagger} S(\rho) M(\rho)=1 .
\end{aligned}
$$

The matrix $M(\infty)$ may be introduced into Eq. (1) and with a little manipulation one obtains

$$
[\bar{H}(\rho)-V(\rho) \bar{S}(\rho)] \bar{C}_{0}(\rho)=0,
$$

where

$$
\begin{aligned}
& \bar{H}(\rho)=M(\infty)^{\dagger} H(\rho) M(\infty)-E_{0}(\infty) \bar{S}(\rho), \\
& \bar{S}(\rho)=M(\infty)^{\dagger} S(\rho) M(\infty), \\
& \bar{C}_{0}(\rho)=M(\infty)^{-1} C_{0}(\rho) .
\end{aligned}
$$

Equation (3) may now be written in partitioned form. ${ }^{10}$ To establish the pattern we display $H$ as

$$
\left[\begin{array}{ll}
H_{G G} & H_{G E} \\
H_{E G} & H_{E E}
\end{array}\right]
$$

with $H_{G G}$ being $1 \times 1$, that is, a single number. $\bar{S}(\rho)$ and $\bar{C}_{0}(\rho)$ are partitioned to match.

Dropping the explicit writing of $\rho$ and eliminating the portion of $\bar{C}$ corresponding to the $E$ subspace, we obtain an implicit equation for $V$,

$$
\begin{aligned}
V= & {\left[H_{G G}-\left(H_{G E}-V S_{G E}\right)\left(H_{E E}-V S_{E E}\right)^{-1}\right.} \\
& \left.\times\left(H_{E G}-V S_{E G}\right)\right] / S_{G G}
\end{aligned}
$$

which, when the second term in the numerator of the righthand side is small enough, may be solved iteratively.

We emphasize that the matrix elements $H(\rho)_{i j}$ and $S(\rho)_{i j}$ are complicated but finite sums over products of various integrals arising from the HF bases used for $a$ and $b$. There are two kinds of integrals. Those involving MOs on only one subsystem may be termed "on board" integrals. Those involving MOs from each of the subsystems will be termed "intersystem integrals," and these are all zero at $\rho=\infty$. Thus $H$ and $S$ may be written as

$$
\begin{aligned}
& H(\rho)=H(\infty)+W(\rho), \\
& S(\rho)=S(\infty)+D(\rho),
\end{aligned}
$$

where, in each case, only on board integrals are in the first term on the right-hand side and all intersystem integrals are in the second. Transforming to $\bar{H}$ and $\bar{S}$, we see that

$$
\begin{aligned}
& \bar{H}(\rho)= E(\infty)-E_{0}(\infty) I \\
&+M(\infty)^{\dagger}\left[W(\rho)-E_{0}(\infty) D(\rho)\right] M(\infty), \\
& \bar{S}(\rho)=I+M(\infty)^{\dagger} D(\rho) M(\infty) .
\end{aligned}
$$

It is clear that in the single element $H_{G G}$ there is an exact cancellation of $E_{0}(\infty)$ and only interaction terms, $D(\rho)$ and $W(\rho)$ are left. We emphasize that this cancellation is not dependent upon how accurately $E_{0}(\infty)$ represents any true values of the molecular energies.

Returning to Eq. (4), we see that, except for the diagonal elements of $H_{E E}-V S_{E E}$, in which the energy differences $E_{i}-E_{0}$ dominate, the numerator consists entirely of quantities dependent upon interactions and hence have the same order of magnitude as $V$. The denominator is 1 plus terms of the interaction type. Thus if the most approximate of the quantities remaining in Eq. (4) is calculated with a percent error $e$, and the CI functions chosen are adequate for calculating $V$, we expect it, also, to have the error $e$, approximately. This is a consequence of having no serious differencing problems remaining explicitly in Eq. (4). Internal to the elements of $W(\rho)$, there remain further cancellations of longrange Coulomb forces between the two subsystems. However, these may be dealt with as discussed below.

As far as algebra is concerned, obtaining the potential from Eq. (4) is exactly equivalent to directly diagonalizing Eq. (1) and using Eq. (2) to obtain the potential. These are not necessarily the same things on a computer, of course, and tests are necessary to establish that the numerical process is not producing errors. This has in fact been done at a number of geometries of our Ne-HF potential discussed in the following paper. In all cases tested, the potential obtained from Eq. (4) was the same as that from Eqs. (1) and (2) to $10^{-9}$ hartrees. Thus our numerical procedures are adequate for calculating these small potentials. Equation (4) is a very useful form for the potential that shows very clearly the expected accuracy as far as matrix elements are concerned. But, these considerations also show that within this VB framework for intermolecular potentials, the conventional procedure of matrix diagonalization and subtraction of $E_{0}(\infty)$ will easily give as many significant figures as are likely to be needed in the potential.

\section{THE HAMILTONIAN AND OVERLAP MATRIX ELEMENTS}

We may obtain another useful idea from an examination of Eq. (4). The submatrix $\left(H_{E E}-V S_{E E}\right)$ occupies a unique place in the expression for $V$. In particular, one would expect that the off-diagonal elements of $H_{E E}$ and $S_{E E}$ are less important than the diagonal elements, and, indeed, in standard perturbation theory they contribute to the energy only in order three and higher. This suggests that we need not calculate these as accurately as we do the others of Eq. (4). We have implemented this idea and in this section we discuss the strategies we use for rapid evaluation of the matrices of $S$ and $H$ in the nonorthogonal VB basis.

For those cases where we use the exact algorithm, the matrix element of the overlap has the form

$$
\begin{aligned}
S(\rho)_{i j} & =\left\langle\phi_{i} \mid \phi_{j}\right\rangle \\
& =\left\langle u_{1}^{i} \cdots u_{n}^{i} \mid A_{a} A_{b}(1-S+D-T+\cdots) u_{1}^{j} \cdots u_{n}^{j}\right\rangle,
\end{aligned}
$$

where $A_{a}$ and $A_{b}$ are antisymmetrizers for $a$ and $b$, respectively, and $S, D, T, \ldots$ are a series of single, double, triple,... interchanges between the two subsystems. The total number of terms in the sum of permutations interchanging electrons 
between the subsystems is the binomial coefficient $\left(\begin{array}{l}n \\ n_{a}\end{array}\right)$ $=\left(\begin{array}{l}n \\ n_{b}\end{array}\right)$, where $n$ is the total number of electrons and $n_{a}$ and $n_{b}$ are the numbers of electrons on $a$ and $b$ separately. The Gaussian elimination method for evaluating determinants requires a length of time proportional to $\mathrm{m}^{3}$ for a determinant of $m$ rows and columns. If this were used on each of the terms of Eq. (6) the total labor would be proportional to

$$
n_{a}^{3} n_{b}^{3}\left(\begin{array}{l}
n \\
n_{a}
\end{array}\right)
$$

a value much larger than that required to evaluate Eq. (5) directly, considered as a single determinant. Even if, as has been suggested ${ }^{11}$ because of the small size of the intersystem overlaps, one includes only the single interchange terms from Eq. (6), the labor of evaluating Eq. (5) directly would be less if $n_{a} n_{b}>\sim 3$. The situation would be even more unfavorable if double interchange terms are required.

Again, we have a situation where numerical truncation on a computer may affect the results. The number of permutations in the term of Eq. (6) for $k$ interchanges is $\left(\begin{array}{c}n_{a} \\ k\end{array}\right)\left(\begin{array}{c}n_{b} \\ k\end{array}\right)$ and if the intersystem overlaps are of the order $\epsilon$, then the $k$ interchanges contribute $\left(\begin{array}{c}n_{a} \\ k\end{array}\right)\left(\begin{array}{c}n_{b} \\ k\end{array}\right) \epsilon^{2 k}$ to the sum. We expect numerical difficulties if this quantity is of the order of unity while $\epsilon^{2 k}$ is $\sim 10^{-16}$ (for a 16 decimal digit computation). This situation cannot occur for a system of, for example, 20 electrons where the largest binomial coefficient product is 63504. In order to verify this conclusion we have actually tested a number of determinants from $\mathrm{Ne}-\mathrm{HF}$ calculations to be reported and have found differences between Eqs. (5) and (6) to occur only in the last significant figure.

The situation for the matrix elements of the Hamiltonian is dominated by the fact that the number of two electron integrals required is proportional approximately to the fourth power of the number of electrons while the work preparatory to obtaining the second order density matrix is still an $n^{3}$ problem. Thus there is again no benefit from evaluating these with expansions of the antisymmetrizer in the manner of Eq. (6).

Therefore, for the matrix elements that we intend to calculate exactly, we use the standard method for evaluating matrix elements between nonorthogonal configurations that one of us has been using for a number of years. For a review of this complete procedure see Gallup et $a l^{12}$

We emphasize that the orbitals we use are orthonormal for each of the subsystems separately. The only nonzero overlaps are from intersystem interactions. Our strategy for the off-diagonal elements of $H_{E E}$ and $S_{E E}$ is to evaluate these as if even the intersystem overlaps were also zero. Thus instead of using a matrix element algorithm for nonorthogonal configurations, we merely use the standard one appropriate for configurations constructed from orthogonal orbitals. This is much faster, of course, and makes it possible to deal effectively with the calculation of large matrices. As the number of configurations increases, the number of matrix elements calculated exactly increases only linearly with the order of the matrices, while the number treated approximately increases with the square of the order. Thus the calculation becomes more efficient as the size of the matrices increases.

\section{THE APPROXIMATION OF THE INTERSYSTEM INTEGRALS}

The cancellation of the errors demonstrated in Sec. II has important consequences for the evaluation of integrals. When using atomic orbital ( $\mathrm{AO}$ ) bases, the principal errors in $a b$ initio calculations arise from inaccuracies in evaluation of the necessary integrals between the AOs and from deficiencies in various aspects of either the one- or $n$-electron bases used. The Gaussian group function representation of AOs deals with the first of these very effectively. The simple analytical properties of Gaussians allow the economical evaluation of integrals to machine accuracy. However, as is well known, Gaussians behave incorrectly at long distances, and particularly for intermolecular forces, this is a basis set deficiency of one type.

Slater functions, on the other hand, have the correct exponential behavior at long distances, but methods of evaluation of some integrals have been unable to attain accuracies better than 6-7 decimal places without using excessive time. ${ }^{13}$ Our analysis suggests that even this accuracy is unnecessarily high for many of the integrals, and that we may use quite approximate methods if proper attention is paid to certain cancellations that must occur in the Coulomb energy. In the next sections we survey the various integrals involved in our procedure and the method we use for evaluation of each of the types that occur.

\section{INTEGRALS FROM POLYCAL}

The Gaussian transform integral package is efficient for all of the overlap and kinetic energy integrals and for the on board nuclear attraction and electronic repulsion integrals. These latter on board integrals are calculated only once for all geometries so even a slower program than POLYCAL ${ }^{13}$ would not be a problem. Indeed, POLYCAL may have its precision parameters set quite high for these integrals.

\section{INTEGRALS BY MULTIPOLE EXPANSIONS}

In our procedure we obtain intersystem nuclear attraction (two orbitals on one subsystem with a nucleus from the other) integrals and intersystem electron repulsion (two orbitals on one subsystem interacting with two orbitals from the other) integrals by a multipole expansion approximation. This is most easily analyzed using the bipolar expansion of $r_{12}^{-1}$, given by Buehler and Hirschfelder. ${ }^{14}$ As is typical for expansions of $r_{12}^{-1}$, a Green's function, different analytic forms are required for different ranges of the variables. Figure 1 shows the four regions labeled A, B, C, and D.

In considering the electron repulsion integrals of the present type, if there were no overlap between the orbitals on the two subsystems, the integral could be obtained exactly by an integration only over region $A$. The same holds for the nuclear attraction integrals, as can be seen by considering a nucleus as a Dirac delta function charge distribution. As the charge distributions approach one another more closely, they will have some overlap, and the other regions of the bipolar expansion, $\mathrm{B}, \mathrm{C}$, and $\mathrm{D}$, will contribute to the result for an exact treatment. We approximate these integrals then, by using only the analytic form appropriate to region $\mathrm{A}$ of 


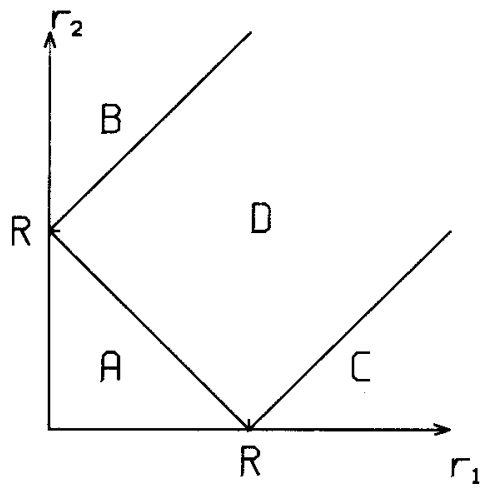

FIG. 1. The regions of $r_{1}-r_{2}$ space for which different analytic expressions are required in the bipolar expansion of $\left(r_{12}\right)^{-1}$.

the bipolar expansion while integrating over all space. Physically, this neglects the exact effects of charge penetration between the two subsystems.

Actually, the nuclear attraction integrals of this type are obtained to fairly high accuracy by a number of techniques. However, Coulomb interactions are quite large and fall off very slowly with distance. For electrically neutral subsystems of ten electrons each, the total repulsion energy at 20 bohr is approximately $10 \mathrm{a}$. $\mathrm{u}$. while the total attraction energy is approximately - 10 a. u. In typical calculations these must very nearly cancel to reach an energy of the order of $10^{-5}$ a.u. It is clear that this will be reliable only if the same technique is used on all integrals of this section.

\section{THE FOURIER TRANSFORM CONVOLUTION THEOREM}

The remainder of the integrals have at least one orbital from each of the subsystems. These may be symbolized as $\left\langle a\left|-Z / r_{b}\right| b\right\rangle$ for nuclear attraction types and $[a a \mid a b]$ or $[a b \mid a b]$ for electron repulsion types. These integrals are all of the exchange type and have no counterpart in the nuclear repulsion part of the energy. In line with the goal of having the correct exponential behavior for these, we chose the method of Fourier transforms. The appropriateness of this choice is seen from an examination of the formulas for the Fourier transform of a two-center distribution that is the product of two Slater orbitals. These have an explicit factor involving the exponential function of the distance between the two orbital centers. An alternative approach might be the application of the bipolar expansion, used for the integrals discussed in Sec. VI. However, this is unsuitable, since all four regions of the bipolar expansion have significant contributions to most of the integrals, and the expansions are expected to converge slowly.

The Fourier transform method has been used extensively in both analytical ${ }^{15}$ and numerical ${ }^{16}$ treatments of two $^{16}$ electron integrals. The principal result relates the Coulomb interaction between two charge distributions $\rho_{a}$ and $\rho_{b}$, to an integral over the product of the Fourier transforms $F_{a}$ and $F_{b}$, respectively,

$$
\iint \rho_{a}(1) \rho_{b}(2) r_{12}^{-1} d \tau_{1} d \tau_{2}=4 \pi \int F_{a} F_{b} d k^{3} / k^{2}
$$

This theorem replaces a sixfold nonseparable integral with three separated threefold integrations.
As a first step one is required to obtain the FT of the product of two orbitals. If these are Slater orbitals at the same center the FT can be evaluated explicitly as a rational polynomial function of $k$ multiplied by spherical harmonics in the angles. The determination of the FT of a Slater orbital product for two different centers has been the subject of a number of studies. ${ }^{17}$ It can easily be reduced to having no more than one numerical integration.

For our procedure we have used a threefold numerical integration over $k$ to approximate these exchange type integrals. This is done in polar coordinates. For the $\phi_{k}$ integration we use a constant coefficient uniformly spaced quadrature formula and for the $\theta_{k}$ integration we use the standard Gauss-Legendre formulas. For the $k$ integration we use a novel quadrature formula designed to improve the convergence by taking advantage of the known asymptotic behavior of the FT for large $|k|$.

For products of Slater orbitals, whether at one center or at two, the dependence of the FT on $k$ is $O\left(k^{-n}\right)$, asymptotically, where $n \geqslant 4$. We make a change of variable

$$
k=y /(1-y)
$$

and with a little manipulation obtain

$$
\int_{0}^{\infty} f(k) d k=\int_{0}^{1} g[y /(1-y)](1-y)^{6} d y,
$$

where $g(k)=f(k) \times(1+k)^{8}$. The function $g$ is bounded in the whole range of $k$ (or $y$ ) and at $y=1$ is either finite or 0 depending upon the power of $k$ in the asymptotic behavior of the FTs. We now obtain the integral on the right-hand side of Eq. (7) by determining the optimal Gaussian type quadrature formula ${ }^{18}$ for the interval 0 to 1 and the weight function $(1-y)^{6}$. This has abscissas that are roots of Jacobi polynomials. ${ }^{19}$ These transformations have the effect of directly putting into the weight function the property of the original integrand that made it converge. Therefore, the quadrature abscissas tend to collect toward the small values of $y$ (and $k$ ) close to 0 where the transforms are larger and the contribution to the integral more important. A report giving more details of this whole procedure will be published elsewhere. ${ }^{20}$

\section{OPTIMIZATION OF EXCITED PSEUDO-STATE ORBITALS FOR CI}

The principal mechanisms for attraction between two closed shell systems are dispersion, induction, and charge transfer. Of these charge transfer presents the greatest problems, and we defer discussion of this phenomenon until later.

If either of the subsystems of our interacting pair possesses an electric multipole moment, both dispersion and induction involve the transition moments of various excitations. Because of the close connection between the polarizability of a system and the $C_{6}$ coefficient of its interaction with other systems we expect that orbitals optimized to obtain the best possible polarizibility will also be optimal for $C_{6}$. In this section we discuss a method for obtaining the most efficient set of excited orbitals for describing the polarizability.

Burke and Robb ${ }^{9}$ have pointed out that the $z z$ component, for example, of the polarizability tensor is the extreme value of the functional

$$
J_{z}=-2\left\langle u\left|H^{(0)}-E_{0}^{(0)}\right| u\right\rangle+4\left\langle u|z| \phi_{0}\right\rangle
$$


with respect to variations in $u$, and that determining the optimum $u$ gives what they call a "pseudo state" to use for introducing the polarizibility into other calculations. For an electric field in the $z$ direction, $u$ is, in other language, the first order correction to the wave function due to the field. The condition that $J_{z}$ be a maximum is easily seen to be

$$
0=-4\left[\left(H^{(0)}-E_{0}^{(0)}\right) u-z \phi_{0}\right]
$$

We now show that a finite field method for polarizibilities is equivalent to the Burke procedure.

Consider the representation of the wave function of a system as a configuration interaction of the Hartree-Fock reference function and single, double, triple,..., excitations that are appropriate for the correlated states of the system. We obtain approximations to the wave functions and energies of these states by diagonalization of the $H$ matrix in this basis:

$$
\bar{H}^{(0)}=U^{\dagger} H^{(0)} U=E^{(0)} .
$$

If now we consider the matrix of the Hamiltonian

$$
H=H^{(0)}-\varepsilon z
$$

in the basis in which $H^{(0)}$ is diagonal, and we write it in partitioned form, we obtain

$$
\begin{aligned}
& H_{00}^{(0)}-\varepsilon z_{00}-E_{0}(\varepsilon)-\varepsilon \sum_{i} z_{0 i} C_{i}=0 . \\
& -\varepsilon z_{0 i}+\sum_{j}\left\{\left[E_{i}^{(0)}(\varepsilon)-E_{0}(\varepsilon)\right] \delta_{i j}-\varepsilon z_{i j}\right\} C_{j}=0 .
\end{aligned}
$$

Equation (12) are the conditions that the functional

$$
\begin{aligned}
K_{z}(u)= & \left\langle u\left|H^{(0)}-\varepsilon z-E_{0}^{(0)}\right| u\right\rangle \\
& -2\left\langle u\left|\varepsilon\left(z-z_{00}\right)\right| \phi_{0}\right\rangle
\end{aligned}
$$

be an extremum when $u$ is expanded in the excited states of Eq. (10). Equation (13) is a slight generalization of Eq. (8) for noncentrosymmetric systems, and the extreme value for $K_{2}$ is

$$
(1 / 2) \alpha \varepsilon^{2}+O\left(\varepsilon^{3}\right) .
$$

Thus determining $\alpha$ from

$$
\alpha=\lim _{\varepsilon \rightarrow 0}-\left(\partial^{2} E_{0}(\varepsilon) / \partial \varepsilon^{2}\right),
$$

where $E_{0}(\varepsilon)$ is obtained by solving the system (11) and (12), is equivalent to the procedure of Burke and Robb minimizing $K_{z}$ within the basis we are using.

We may use a somewhat more approximate treatment to obtain a set of optimal excited orbitals. If, instead of the solutions of Eq. (10), we solve Eqs. (11) and (12) using only the Hartree-Fock function and functions formed by single excitations from it into the virtual orbitals, the resulting first order wave function can be written in terms of single excitations into a new set of orbitals. The lowest eigenfunction of Eqs. (11) and (12) can be written

$$
\psi=\phi_{0}+\sum_{i j} \phi_{i}^{j} C_{i j},
$$

where $\phi_{0}$ is the Hartree-Fock function and $\phi_{i}^{j}$ is the singlet function resulting from an excitation of the occupied orbital $u_{i}$ into the virtual orbital $v_{j}$. The orbitals obtained as the linear combinations

$$
v_{i}^{\prime}=\sum_{j} C_{i j} v_{j}
$$

may be subjected to a Schmidt (or other) orthogonalization, and the orthonormal functions $w_{i}$ that result constitute the optimal set we have been seeking. In general, for $\phi_{0}$ with $n$ doubly occupied orbitals, we obtain $n$ optimal excited orbitals, and $\psi$ may be written as the HF function plus $n^{2}$ single excitations. Systems with high symmetry allow a more economical representation than this.

We repeat the process for the $x$ and $y$ directions and in this way obtain a collection of optimal excited orbitals that, with the HF function, give the most economical representation of the wave function for a field component in any direction.

\section{A LEVEL SHIFTING CORRECTION FOR POLARIZABILITIES}

Unless the treatment reproduces the polarizability correctly, a calculation of the intermolecular potential between two systems cannot be expected to be accurate. The well known formula for the polarizability, derived by means of perturbation theory, is

$$
\alpha_{z z}=2 \sum_{i}\left|z_{0 i}\right|^{2} /\left[E_{i}^{(0)}-E_{0}^{(0)}\right]
$$

for the $\alpha_{z z}$ component of the tensor. The procedure we use to obtain the optimal pseudo-orbitals still generally underestimates the polarizability for closed shell systems. Since for the ground state all the terms of Eq. (14) are positive, this implies that the HF function gives transition moments that are too small, excitation energies that are too large, or both. In a finite basis calculation the sum is also truncated. We expect that a change in the excitation energies will increase the calculated polarizability to the correct value. This is done within the finite field method by modifying Eq. (12) with a correction in the diagonal elements

$$
-\varepsilon z_{i 0}+\sum_{j}\left\{H_{i j}^{(0)}-\varepsilon z_{i j}+\left[\Delta-E_{0}(\varepsilon)\right] \delta_{i j}\right\} C_{j}=0
$$

and then adjusting $\Delta$ to give the correct polarizibility.

The dispersion in an intermolecular potential calculation must also be treated accurately for good results. We have shown ${ }^{21}$ that the level shift corrections for the polarizability for several systems can be used additively to correct the value of the $C_{6}$ coefficient obtained using the same basis. For more details we refer the reader to the other article.

This level shifting correction is easily carried out in the context of a CI calculation of intermolecular forces. We are thus able to introduce some independent semiempirical information into our calculation, without having to deal with damping factors, which introduce so much uncertainty into some treatments.

\section{CHOICE OF CONFIGURATIONS FOR THE CI}

In the previous sections of this article we have concentrated upon methods of approximating various integrals and matrix elements. In this section we will examine some points about the physics of the interactions and the way the various 
terms in the wave function describe them.

If the subsystems both possess electric moments, the multipole interactions between these will be described by the product of the two HF functions. This will still be true even if a more general function were to be used for the "ground state." In many cases the multipole interaction will be the dominant one. If either of the subsystems is monotomic there are no multipolar interactions between them represented in the ground state function, and the interaction is purely repulsive.

Whether or not multipole interactions are present, the remainder of the potential arises from terms involving excited configurations. Consequently it is important to include the appropriate excited states in the CI matrix. In general it is necessary to include three distinct classes of excitation ${ }^{22}$; and these are examined in turn below.

\section{A. Polarization}

Also called induction, this phenomenon is relatively unimportant for atomic systems, but it can be sizable when one or both of the subsystems possesses an electric moment. When one of the moments is a dipole, polarization leads to terms in the potential that vary asymptotically with the internuclear distance as $R^{-6}$. This effect then combines with the dispersion, (see below) which has the same form. Single excitations out of the ground state function represent polarization. It appears to be a relatively uncomplicated phenomenon that, nevertheless, requires good values for the subsystem polarizabilities for its correct reproduction, as was emphasized earlier.

\section{B. Dispersion}

It has long been known that this phenomenon is essentially an intermolecular electron correlation effect. Some workers have described it in terms of emission and absorption of virtual photons. ${ }^{23}$ The necessary configurations to represent it consist of double excitations, one on each of the subsystems. The asymptotic form of the dispersion interaction is a sum of powers of $R^{-n}, n \geqslant 6$. If both of the subsystems are centrosymmetric only even inverse powers of $R$ are present.

\section{Charge transfer}

The role of charge transfer in intermolecular forces has been difficult to assess. In VB wave functions it can give rise to basis set superposition errors as well as contributing to any real physical effects present. These errors can lead to considerable overestimates of the interaction energy. ${ }^{24}$ The size of the superposition error is quite sensitive to the quality of the ground state functions and to the optimization of the orbitals used in the excited configurations. ${ }^{3,7}$ For relatively small systems with large ionization potentials one might argue that we may neglect this type of interaction. For large systems it can be important. ${ }^{25}$

\section{SUMMARY AND CONCLUSION}

In this article we present a method for the direct calculation of intermolecular potential energy surfaces. The ap- proach uses wave functions determined from the individual subsystems in the construction of the wave function for the composite system, which in turn is determined by a variation-perturbation approach based upon VB theory. We implement the procedure with a systematic series of approximations that are justified by the physical situation, i.e., that the energy of interaction is small (of the order of $10^{-5} \mathrm{a}$. u.), that the overlap between the subsystems is small-but not negligible, and that the separation between the systems is relatively large.

Our procedure may be summarized as follows: In order to construct the matrix

$$
H-S E \text {, }
$$

(1) Calculate the diagonal elements $H_{i i}$ and the elements of the first row and column $H_{i 1}, H_{1 i}, S_{i 1}$, and $S_{1 i}$ exactly. Other elements $H_{i j}$ and $S_{i j}$ are approximated by neglecting overlap between the subsystems (Sec. III).

(2) Determine the optimized excited orbitals by pseudostate technique (Sec. VIII).

(3) Introduce level shifts to correct subsystem polarizabilities (Sec. IX).

(4) Approximate intersystem integrals:

(i) Integrals $\left[a a^{\prime} \mid r_{b}^{-1}\right],\left[b b^{\prime} \mid r_{a}^{-1}\right]$, and $\left[a a^{\prime} \mid b b^{\prime}\right]$ determined from multipole expansions (Sec. VI).

(ii) Integrals $\left[a b \mid r_{a}^{-1}\right],\left[a b \mid r_{b}^{-1}\right],\left[a a^{\prime} \mid a b\right]$, $\left[b b^{\prime} \mid a b\right]$, and $\left[a b \mid a^{\prime} b^{\prime}\right]$ approximated using Fourier transform method (Sec. VII).

(iii) Calculate the remaining integrals using POLYCAL, Gaussian-transform method for Slater orbitals (Sec. V).

In the article following this one, we apply our procedure to the case of Ne-HF. For such a system our method involves approximations of many kinds, including those made in tens of thousands of integrals. We feel that the only real test of results using it can be a comparison with an experimental potential of high quality. In this way we can test the adequacy of the concerted use of all these approximations.

Nevertheless, many of the approximate techniques for evaluation of integrals and matrix elements can undoubtedly be improved to make the procedure even more accurate and efficient. Work continues on these questions.

'R. Eisenschitz and F. London, Z. Phys. 60, 491 (1930); J. O. Hirschfelder and R. Silbey, J. Chem. Phys. 45, 2188 (1966); J. N. Murrell and G. Shaw, ibid. 46, 1768 (1967); J. I. Musher and A. T. Amos, Phys. Rev. 164, 31 (1967); J. O. Hirschfelder, Chem. Phys. Lett. 1, 363 (1967); A. Van der Avoird, J. Chem. Phys. 47, 3649 (1967); D. M. Chipman, ibid. 66, 1830 (1977).

${ }^{2}$ R. Peierls, Proc. R. Soc. London Ser. A 333, 157 (1973); B. Atalay, A. Mann, and R. Peierls, ibid. 335, 251 (1973).

${ }^{3}$ G. A. Gallup, Mol. Phys. 33, 943 (1977); J. Schaefer and W. Meyer, J. Chem. Phys. 70, 344 (1979); W. Meyer, P. C. Hariharan, and W. Kutzelnigg, ibid. 73, 1880 (1980); B. Liu and A. D. MacLean, ibid. 59, 4557 (1973); M. Raimondi, Mol. Phys. 53, 161 (1984).

${ }^{4}$ R. Schinke, H. Meyer, U. Buck, and G. H. F. Dierckson, J. Chem. Phys. 80, 5518 (1984)

${ }^{5}$ S. F. Boys and F. Bernardi, Mol. Phys. 19, 553 (1970).

${ }^{6}$ P. W. Fowler and A. D. Buckingham, Mol. Phys. 50, 1349 (1983).

7P. E. S. Wormer and A. Van der Avoird, J. Chem. Phys. 62, 3326 (1975);

J. M. Norbeck, P. R. Certain, and K. T. Tang, J. Chem. Phys. 63, 590 (1975); J. Gerratt, Proc. R. Soc. London Ser. A 350, 363 (1976); G. A. Gallup, J. Chem. Phys. 66, 2252 (1977); P. Cremaschi, G. Morosi, M. Rai- 
mondi, and M. Simonetta, Mol. Phys. 38, 1555 (1979); J. Gerratt and M. Papadopoulas, ibid. 41, 1071 (1980); G. A. Gallup, ibid. 49, 865 (1983); J.

R. Collins and G. A. Gallup, ibid. 49, 871 (1983).

${ }^{8}$ V. K. Lan, J. Phys. B 5, 242 (1972).

${ }^{9}$ P. G. Burke and W. D. Robb, Adv. At. Mol. Phys. 11, 144 (1975), Sec. III. 10P.-O. Lowdin, J. Mol. Spectrosc. 13, 326 (1964).

"J. Gerratt, Proc. R. Soc. London Ser. A 350, 363 (1976).

${ }^{12}$ G. A. Gallup, R. L. Vance, J. R. Collins, and J. M. Norbeck, Adv. Quantum Chem. 16, 229 (1982).

${ }^{13}$ POLYCAL, multicenter integrals over Slater orbitals by the Gaussian transform method, R. M. Stevens, Harvard University; I. Shavitt and M. Karplus, J. Chem. Phys. 36, 550 (1962); 1. Shavitt, in Methods of Computational Physics, edited by B. Alder, S. Fernbach, and M. Rotenberg (Academic, New York, 1963), Vol. 2, p. 1.

${ }^{14}$ R. J. Buehler and J. O. Hirschfelder, Phys. Rev. 83, 628 (1953); see also B. C. Carlson and G. S. Rushbrook, Proc. Cambridge Philos. Soc. 46, 626 (1950).

${ }^{15}$ R. A. Bonham, J. L. Peacher, and H. L. Cox, J. Chem. Phys. 40, 3083 (1964); H. J. Silverstone, ibid. 48, 4098, 4106, 4108 (1968); K. G. Kay and
H. J. Silverstone, ibid. 51, 956, 4287 (1969); 53, 4269 (1970); H. J. Monkhorst and F. E. Harris, Int. J. Quantum Chem. 6, 601 (1972).

${ }^{16}$ A. Graovac, K. Kovačević, Z. B. Maksić, and A. Veseli, Int. J. Quantum Chem. 17, 747 (1980).

${ }^{17}$ D. F. Gallaher and A. Msezone, J. Phys. B 7, 127 (1974); 8, 112 (1975); B. R. Junker, 13, 1049 (1980).

${ }^{18}$ V. I. Krylov, Numerical Approximation of Integrals (MacMillan, New York, 1962).

${ }^{19}$ M. Abramowitz and I. A. Stegun, Handbook of Mathematical Functions (Dover, New York, 1965).

${ }^{20} \mathrm{G}$. A. Gallup and J. Gerratt, J. Comput. Chem. (to be submitted).

${ }^{21}$ G. A. Gallup and J. Gerratt, Chem. Phys. Lett. 112, 228 (1984).

${ }^{22} \mathrm{H}$. Margenau and N. R. Kestner, Theory of Intermolecular Forces, 2 nd ed. (Pergamon, New York, 1969).

${ }^{23}$ D. P. Craig, Adv. Quantum Chem. 16, 198 (1982).

${ }^{24}$ D. Chen, J. R. Collins, and G. A. Gallup, J. Chem. Phys. (submitted for publication).

${ }^{25}$ R. Foster, Organic Charge-Transfer Complexes (Academic, New York, 1969), see Sec. 9A. 